\title{
Homogeneous Fibroglandular Echotexture
}

National Cancer Institute

\section{Source}

National Cancer Institute. Homogeneous Fibroglandular Echotexture. NCI Thesaurus.

Code C69213.

A description of homogeneous fibroglandular elements seen in a tissue composition image obtained by sonography. 